\title{
WIND DRIVEN MASS TRANSFER IN INTERACTING BINARIES
}

\section{CHRISTOPHER A. TOUT AND DOUGLAS S. HALL \\ Lick Observatory \\ University of California \\ Santa Cruz \\ Dyer Observatory \\ Vanderbilt University \\ Nashville}

CA 95064

U.S.A.
Tennessee 37235

U.S.A.

\begin{abstract}
ABSTR.ACT. Stars in close binary systems can suffer two kinds of mass change: 1) mass transfer between the stars 2) mass loss completely from the system. Observational estimates indicate that these are of the same order. A simple explanation can be found if the mass loss, by stellar wind, from the Roche-filling star is the driving mechanism behind mass transfer. We find quantitative estimates for the necessary conditions and find that the mass transfer rate and the mass loss rate are indeed similar. We find that the radii of evolved semi-detached systems are more consistent with wind-driven evolution than the traditional nuclear-driven Roche-lobe over flow.
\end{abstract}

\section{Introduction}

The origin of this idea was the realization that the evolved star in a binary prior to Rochelobe overflow (RLOF) appears to be losing mass at a rate comparable to, actually faster than, the rate at which it was thought to lose by mass transfer once it had become a semidetached binary, after RLOF. In a semi-detached binary undergoing conservative case $B$ mass transfer, after passing the critical mass ratio (defined later), the loser would lose mass on its nuclear timescale. Since

$$
\frac{M}{\dot{M}} \approx \tau_{n u c} \approx 10^{11} \frac{M}{L} \text { years, }
$$

where $M$, the mass of the star, and $L$, its luminosity, are in solar units, we would get $\dot{M} \approx 5 \times 10^{-11} M_{\odot} \mathrm{yr}^{-1}$ for a typical late-type subgiant or $\dot{M} \approx 2 \times 10^{-10} M_{\odot} \mathrm{yr}^{-1}$ for a typical late-type giant. There exist both theoretical (Tout and Eggleton 1988) and observational (Mullen et al. 1989) indications that the evolved star in a binary, just before becoming a semi-detached Algol-type system, is losing mass in an enhanced wind at a rate of $10^{-8} M_{\odot} \mathrm{yr}^{-1}$ (if a subgiant) or $10^{-7} M_{\odot} \mathrm{yr}^{-1}$ (if a giant). Such rates are two or three orders of magnitude greater than the conservative case $B$ nuclear rates would be. We suspected that such rapid rates, if taken into account, would alter our understanding of Algol evolution profoundly. In fact the enhanced wind can dominate and, moreover, drive the mass transfer throughout the semi-detached phase of the binary's lifetime. 


\section{Red Giant Stars and the Critical Mass Ratio}

If the lobe-filling star is a red giant we can find a simple expression for its radius. By constructing detailed models of red giant stars Eggleton and Tout (Tout 1989) found an empirical fit to red giant radii in terms of their total mass and luminosity:

$$
R=f(L) M_{1}^{n}
$$

with $n=-0.27$. The suffix 1 or no suffix refers to the mass-losing star while the suffix 2 refers to the gainer. Paczyński (1965) showed that the radius responds to mass loss on a dynamical timescale. If mass transfer in such a system were to result in the Roche lobe shrinking with respect to the losing star, positive feedback would force overflow to accelerate on a dynamical timescale. To avoid this any mass transfer must result in the Roche-lobe radius, $R_{L}$, growing faster than $R$ or $\dot{R}<\dot{R}_{L}$ while $R=R_{L}$. Differentiating Pacyński's (1971) formula,

$$
\frac{R_{L}}{d}=\frac{2}{3^{\frac{4}{3}}}\left(\frac{M_{1}}{M}\right)^{\frac{1}{3}}, \quad 0<q<0.8,
$$

which is actually a good fit to $q \approx 3,(M$ is the system mass and $d$ its semi-major axis) while conserving the total mass and combining these with the total angular momentum, $J \propto M_{1} M_{2} \sqrt{d / M}$, in the case of conservative mass transfer $\left(\dot{M}=\dot{J}=0, \dot{M}_{2}=-\dot{M}_{1}\right.$, throughout $-\dot{M}$ corresponds to the wind-mass-loss rate and $\dot{M}_{2}$ to the mass-transfer rate, $\dot{M}_{1}=\dot{M}-\dot{M}_{2}$ ) requires a mass ratio,

$$
q<\frac{5+3 n}{6}=q_{\mathrm{crit}}
$$

or for red giants $q<0.7$.

\section{Wind Driven Transfer}

If $q<q_{\text {crit }}$ is satisfied, a system can be maintained in a semi-detached state either by expansion on a nuclear timescale or by wind mass loss from the primary coupled with its own expansion following the loss of mass. Consider a Roche-filling $\operatorname{star}\left(R=R_{L}\right)$. It will fall out of its semi-detached state if $\dot{R}<\dot{R}_{L}$ when $\dot{M}_{2}=0$ (since transfer ceases if $R<R_{L}$ ). Consider a case where there is no extra angular momentum loss so that $\dot{J}=\dot{M} d_{1}^{2} \Omega$. With equation (2), $\dot{R}<\dot{R}_{L}$ is satisfied if

$$
\frac{n \dot{M}}{M_{1}}<\frac{M-4 M_{1}}{3 M_{1} M} \dot{M}
$$

so for $n>-1$, windy mass loss will maintain the Roche filling state and thus drive mass transfer if

$$
q<\frac{1-3 n}{3(n+1)}
$$

which holds for $q<0.826$ if $n=-0.27$ (i.e. for giants). On the other hand it cannot be satisfied for $n>\frac{1}{3}$ providing a cut-off on wind driven transfer preventing it from taking 
place for any star that shrinks fast enough. In addition the mass-loss timescale must be much less than the nuclear timescale if wind-driven transfer is to dominate.

If these conditions are satisfied then loss of mass from the primary will keep the system semi-detached so that, with $\dot{M}<0$ and $\dot{M}_{2}>0, R=R_{L}$ and $\dot{R}=\dot{R}_{L}$

$$
\dot{M}_{2}=\frac{3(1+n) q+3 n-1}{6\left(1-q^{2}\right)+(3 n-1)(q+1)} \dot{M}
$$

As expected conditions (4) and (6) must be satisfied if $\dot{M}_{2}$ is to have the opposite sign to $\dot{M}$ and transfer is in the right direction.

\section{Numerical Models}

Numerically we can take into account more of the details. In particular we can use the true Roche-lobe radius and we can easily vary the Alfvén radius so that the wind can carry off additional angular momentum. In addition we can include nuclear evolution for a red-giant loser.
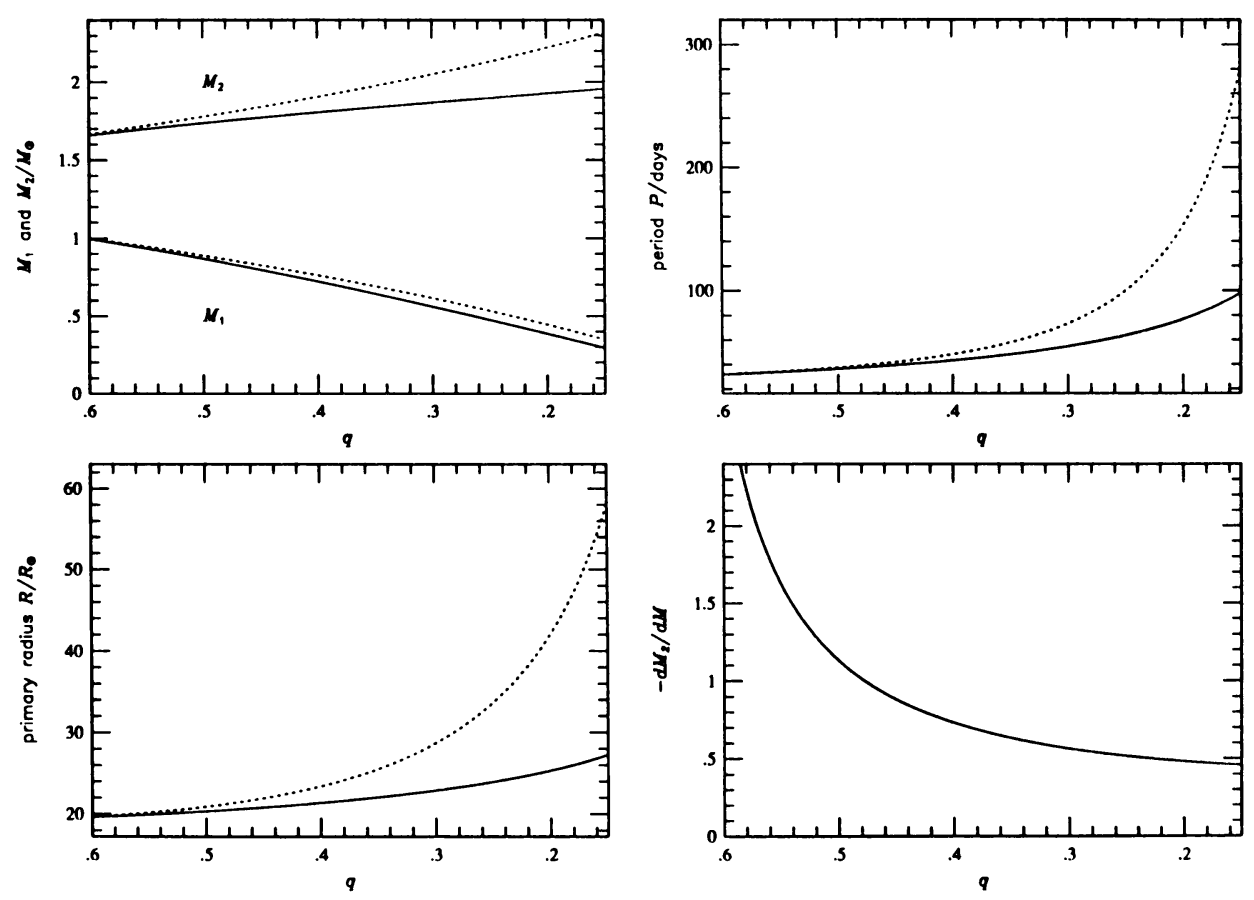

Figure 1. Wind driven transfer - observable parameters as functions of the mass ratio. The solid line is the wind driven case $\left(\dot{M}=-10^{-5} M_{\odot} \mathrm{yr}^{-1}\right)$ and the dotted line is the nuclear driven case $(\dot{M}=0)$. The last graph shows the ratio of the mass transfer rate to the mass loss rate. 
Figure 1 shows the evolution of a system in which $\dot{M}=10^{-5} M_{\odot} \mathrm{yr}^{-1}$ (solid lines). Initially it has $M_{1}=1 M_{\odot}, q=0.6$ and $L=100 L_{\odot}$. In the first three panels we have plotted the variation of the masses, the period and the radius of the primary against the mass ratio. The fourth panel shows the ratio of the mass-transfer rate to the wind-loss rate, $-\dot{M}_{2} / \dot{M}$. We have also plotted, in the first three panels, the same initial system evolved with $\dot{M}=0$ (dotted lines). In this case the evolution is driven by the nuclear burning of the primary. Notice that $P$ and $R$ increase by substantially more over the same range of $q$. However, it is important to realize that the timescale is about $10^{3}$ times longer than that of the wind driven case and so $P$ and $R$ would actually be increasing at a slower rate. If the timescales for nuclear burning and mass loss are comparable then the evolution will lie somewhere between the dotted and solid curves.

\section{Observational Consequences: the Increase in Radius}

A way of using observation to verify the correctness of our wind-driven mass-transfer theory is to examine the increase of radius, $R$, with decreasing mass ratio, $q$. Conservative masstransfer theory, the dotted curve in the radius-versus- $q$ plot in figure 1 , predicts a dramatic increase in radius. From $q=q_{\text {crit }}$ to $q=0.1$ there is a fivefold increase. Our wind-driven mass-transfer theory predicts a much less dramatic increase, the solid line in the same plot of figure 1. From $q=q_{\text {crit }}$ to $q=0.1$ the radius increases by only a factor of 1.7 . With additional angular momentum loss the increase is even smaller because the primary does not have to lose so much mass to achieve the same mass ratio.

As an observational test (amplified in Tout and Hall 1991) we consider known semidetached binaries with the evolved star late enough in spectral type to be convective and find their radii to be consistent with the smaller increase predicted by wind-driven mass transfer.

\section{Acknowledgments}

CAT is most grateful to Emmanuel college, the Institute of Astronomy and the organizers of the 107th IAU Colloquium on Algols for their support of his attendance at that conference where these ideas were conceived during an evening's socializing and to the UK SERC for making possible this visit to Córdoba.

\section{References}

Mullen, D. J., Sion, E. M., Bruhweiler, F. C. and Carpenter, K. G., 1989. Astrophys. J., 339, L33.

Paczyński, B., 1965. Acta astr., 15, 89.

Paczyński, B., 1971. Ann. Rev. Astr. Astrophys., 9, 183.

Tout, C. A., 1989. Phd. Dissertation, University of Cambridge.

Tout, C. A. and Eggleton, P. P., 1988. Mon. Not. R. astr. Soc., 231, 823.

Tout, C. A. and Hall, D. S., 1991. Mon. Not. R. astr. Soc., in press. 